\title{
The effects of testosterone and oestrogen on gonadectomised and intact male rat anterior pituitary mitotic and apoptotic activity
}

\author{
L A Nolan and A Levy \\ Henry Wellcome Laboratories for Integrative Neuroscience and Endocrinology, University of Bristol, Dorothy Hodgkin Building, Whitson Street, \\ Bristol BS1 3NY, UK \\ (Requests for offprints should be addressed to L A Nolan; Email: lesley.a.nolan@bris.ac.uk)
}

\begin{abstract}
We have used a direct, non-immunochemical and highly accurate method to quantify the effects of testosterone and oestrogen on mitotic and apoptotic activity in the young, male rat anterior pituitary in vivo. Surgical gonadectomy resulted in a 3-fold increase in mitotic activity by the fourth post-operative day, which returned gradually to levels seen in intact animals over the subsequent 3-4 weeks. Both a single dose of Sustanon, a mixture of long-acting testosterone esters in arachis oil, and the same dose divided over 7 days (starting 6 days after gonadectomy), initially suppressed mitotic activity to levels seen in intact animals, but was associated after 48-96 h with a wave of increased mitotic activity. The latter was blocked by co-administration of Sustanon with the non-steroidal aromatase inhibitor letrozole and was not seen when the non-aromatisable androgen dihydrotestosterone was
\end{abstract}

substituted for Sustanon. Oestrogen alone in gonadectomised and intact rats produced a marked increase in mitosis as expected. With the exception of a transient increase in response to a single high-dose injection of Sustanon in gonadectomised animals, apoptotic activity was unaffected by all of the above. This study suggests that pituitary mitotic activity is tonically inhibited by gonadal hormone production (at least in the short term) in adult male rats. The study also suggests that supraphysiological testosterone treatment - while unable to reduce anterior pituitary mitotic activity in untreated, intact animals suppresses the early increase in mitotic activity induced by gonadectomy. Oestrogen, either exogenous or generated locally by aromatisation, stimulates anterior pituitary mitotic activity in a time-dependent manner.

Journal of Endocrinology (2006) 188, 387-396

\section{Introduction}

Variations in physiological demands on the hypothalamopituitary-gonadal axis during development and throughout adulthood produce some of the most profound central neuroendocrine changes seen. At the pituitary level these may be manifest not only as alterations in patterns of hormone secretion but also, to some extent, as transient fluctuations in rates of anterior pituitary mitotic and apoptotic activity. Whether changes in overall pituitary size relate predominantly to enhanced vascularity, increased average cell size or an expanded number of parenchymal cells is often unclear, but remodelling of cell populations through transient alterations in the balance between mitotic activity and apoptotic activity may potentially produce changes that persist. Variations in mitotic and apoptotic activity in the anterior pituitary in response to transient but repeated hormonal stimuli are of considerable interest in defining not only potential drifts in physiological responsiveness to recurrent stimuli but also pathophysiological events that may contribute to the induction and/or propagation of pituitary adenomas. The high prevalence of pituitary microadenomas
(Molitch 1997), and the absence of typical phenotypic and genotypic characteristics of malignancies seen in other organ systems, raises the possibility that in some cases these tumours may result from an exuberant but essentially physiological trophic response that fails to resolve after one or particularly recurrent stimuli that transiently uncouple mitotic and apoptotic activity have passed (Levy 2000, Levy \& Lightman 2003).

The gonadotroph lineage is one of the most frequently implicated factors in pituitary adenoma formation, yet patterns of trophic responses even under baseline conditions remain unclear. In males, testicular androgens are clearly the principle hormonal mediators of the feedback loops acting either indirectly via hypothalamic sites and/or directly via the pituitary to control circulating levels of gonadotrophins. As they are subject to aromatisation to oestrogen and 5-alpha reductase conversion to dihydrotestosterone (DHT), the putative increase in mitotic gonadotrophs following castration (Sakuma et al. 1984, Inoue et al. 1985) may be mediated via a number of receptor types and associated signalling pathways (Stefaneanu 1997, Lindzey et al. 1998, Pelletier 2000). 
In order to define more precisely how circulating gonadal hormones modify both cumulative and summative changes in overall pituitary trophic (i.e. mitotic and apoptotic) activity, in the present study we have carefully examined the responses of the rat anterior pituitary to surgical gonadectomy and various sex hormone treatments over a period of 4 weeks. To do this we have used a well-validated manual, but computer-assisted, image analysis system to quantify directly identified mitotic and apoptotic events in thin haematoxylin- and eosin-stained anterior pituitary sections (Nolan \& Levy 2001, 2003, Nolan et al. 2004a, 2004b). The data have allowed us to build a more complete portfolio of understanding of trophic influences on the male rat pituitary (Levy 2002).

\section{Materials and Methods}

All procedures were carried out in accordance with UK Home Office animal welfare regulations. Male Wistar rats weighing 125-150 g were purchased from Bantin and Kingman Universal Ltd (Hull, UK), and were allowed to acclimatise for 1 week in the animal holding facility before being surgically gonadectomised under $1 \mathrm{ml}$ per $100 \mathrm{~g}$ body weight Avertin anaesthetic (a mixture of 2:2:2 tribromoethanol $(2 \% \mathrm{w} / \mathrm{v})$ in $100 \%$ ethanol $(8 \% \mathrm{v} / \mathrm{v})$ and 2-methylbutanol-2-ol $(1 \cdot 2 \% \mathrm{v} / \mathrm{v})$ in $0.9 \%$ saline). For post-operative pain relief, rats were given a s.c. injection of the non-steroidal anti-inflammatory Carprofen (Pfizer, Kent, UK) $4 \mathrm{mg} / \mathrm{kg}$ body weight in a total volume of $0 \cdot 2 \mathrm{ml}$ diluted in saline before recovery from anaesthetic. Groups of rats were killed at intervals up to 28 days after surgery.

At either 6 or 28 days after surgery, further groups of gonadectomised, sham-operated or control intact rats received one of the following supraphysiological hormone treatments:

1. A single s.c. injection of Sustanon $(250 \mathrm{mg} / \mathrm{kg}$ body weight; Sustanon 250 is a long-acting mixture of testosterone esters - testosterone propionate (20\%), testosterone phenylpropionate $(40 \%)$ and testosterone isocaproate (40\%)) (Organon Laboratories Ltd, Cambridge, UK) diluted to $200 \mu \mathrm{l}$ in arachis oil (Sigma; product no. P2144). Control groups of gonadectomised or intact rats were given arachis oil vehicle only.

2. Daily s.c. injections of Sustanon $(35.7 \mathrm{mg} / \mathrm{kg}$ body weight diluted to $200 \mu \mathrm{l}$ in arachis oil) for up to 7 days with control groups of gonadectomised or intact rats receiving arachis oil vehicle only.

3. Daily s.c. injections of DHT $5 \mathrm{mg} / \mathrm{kg}$ body weight ( $5 \alpha$-androstan-17 $\alpha$-ol-3-one (Sigma; product no. A-8380) in $200 \mu \mathrm{l}$ sesame oil) for up to 7 days with control groups of gonadectomised or intact rats receiving sesame oil vehicle only.

4. Daily s.c. injections of oestradiol $(5 \mathrm{mg} / \mathrm{kg}$ body weight $\beta$-oestradiol (Sigma; product no. P-2144) in $200 \mu \mathrm{l}$ sesame oil) for up to 7 days. Control groups of gonadectomised or intact rats were given sesame oil vehicle only.

5. Daily s.c. injections of letrozole $(1 \mathrm{mg} / \mathrm{kg}$ body weight finely suspended but not dissolved in $200 \mu \mathrm{l} 0.3 \%$ hydroxypropyl cellulose (molecular mass $370000 \mathrm{Da}$; Sigma-Aldrich product no. 191892)) for up to 10 days beginning 4 days after gonadectomy, together with $35.7 \mathrm{mg} / \mathrm{kg}$ body weight Sustanon daily beginning 2 days later for up to 7 days. Control groups of gonadectomised rats were given letrozole together with sesame oil vehicle or Sustanon together with hydroxypropylcellulose vehicle. Groups of intact control animals were given both vehicles.

\section{Preparation of tissue sections}

Immediately after decapitation, pituitary glands were carefully removed and fixed in 4\% formaldehyde in PBS for $48 \mathrm{~h}$. The tissue was then washed in two changes of fresh PBS before being embedded in 1\% agar and processed for paraffin wax embedding. A series of $2 \mu \mathrm{m}$ thick axial sections were cut from each pituitary for trophic analysis.

\section{Image analysis for trophic activity}

Apoptotic and mitotic event prevalence was analysed on $2 \mu \mathrm{m}$-thick haematoxylin- and eosin-stained pituitary sections at $\times 1000$ magnification (Nolan et al. 1998). The dedicated real-time computer system used (AxioHOME, Zeiss (Brugal et al. 1992)) projects a virtual image of a computer screen fractionally above the histological section. Different identifier tags manually placed over normal, mitotic or apoptotic cells remain in registration with the targets irrespective of subsequent stage movements and magnification changes. The system retains a cumulative score of the numbers of each cell type counted thus permitting accurate quantification for each section studied. For each animal, three random areas of approximately $47000 \mu \mathrm{m}^{2}$ were scored. By defining area boundaries at low power and then counting events at high power, selection bias and double scoring were eliminated, allowing the error in quantifying the number of normal cells surrounding these events to be $<2 \%$. The sensitivity of detection of counting trophic events throughout the study was virtually $100 \%$, and thus the overall error in estimating the prevalence of trophic events was approximately $0 \cdot 001 \%$.

The histological characteristics used to define apoptotic cells were clusters of two or more extremely dense, round or oval structures varying in diameter from approximately 0.7 to $4 \mu \mathrm{m}$ and surrounded by normal cells (Nolan et al. 2003). Earlier stages of apoptosis cannot be visualised using haematoxylin and eosin staining and light microscopy.

All slides were coded and counted by one blinded observer (LA N) and results expressed as a percentage of the total cell numbers counted for each animal. 


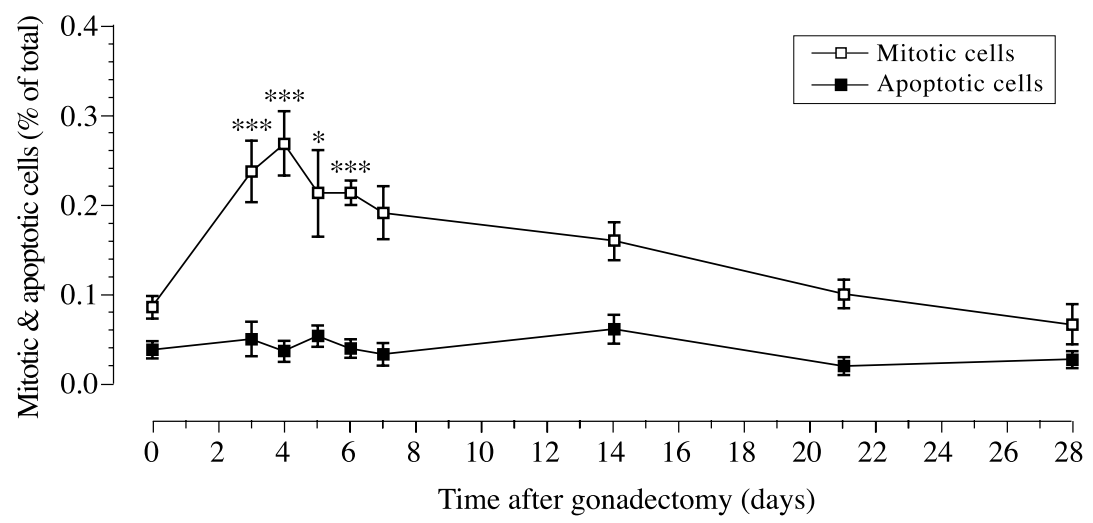

Figure 1 Changes in the prevalence of mitotic figures and apoptotic bodies as a percentage of total parenchymal cells following surgical gonadectomy at time zero. Means \pm s.E. are shown. ${ }^{* *} P<0.001$ and ${ }^{*} P<0.05$ compared with control gonadal-intact animals ( $n=6$ at all time points).

\section{Statistics}

Data were expressed as the mean \pm S.E. GraphPad Prism (GraphPad Software, San Diego, CA, USA) was used to perform statistical calculations. Differences between groups were evaluated using one-way ANOVA followed by Tukey-Kramer multiple comparison post-hoc tests. $P<0 \cdot 05$ was considered statistically significant.

\section{Results}

The effects of gonadal status on anterior pituitary mitotic and apoptotic activity

In intact, young adult male control rats, the prevalence of directly observed mitotic figures and apoptotic figures in the anterior pituitary was $0.086 \pm 0.01$ and $0.036 \pm$ $0 \cdot 008 \%$ respectively (Fig. 1). This apparent discrepancy in the baseline prevalence of mitotic figures versus apoptotic bodies in control animals is an artefact of the method of detection. As mitosis and apoptosis must be in absolute balance or differ by an undetectably small amount associated with growth, using the present methods of histological identification mitotic figures are visible for 2-3 times longer than apoptotic bodies (Nolan et al. 1998).

Between 3 and 6 days after surgical gonadectomy, the prevalence of mitotic figures remained significantly higher than in intact control animals and reached a peak 4 days after surgery $(0 \cdot 269 \pm 0 \cdot 036 \% ; n=6 ; P<0 \cdot 001$ compared with intact controls) before gradually returning to baseline levels by 28 days after surgery (Fig. 1). At 4 days after surgery, the mitotic index in sham-operated animals was $0 \cdot 089 \pm 0 \cdot 01 \%(n=6$; data not shown $)$, a level not significantly different from intact, untreated controls. No concurrent changes in the prevalence of apoptotic bodies were detected throughout the whole experimental period (Fig. 1). In absolute terms, as the total number of mitotic and apoptotic figures remains low at a fraction of one per cent in these and all following experiments, it was not possible to correlate trophic events with different cell types identified immunocytochemically with sufficient statistical integrity to be useful conceptually.

Trophic effects of testosterone and oestrogen treatments - the influence of time and dose

A single bolus of long-acting testosterone esters (Sustanon) given 6 days after gonadectomy resulted in a significant fall in the anterior pituitary mitotic index within $24 \mathrm{~h}$, followed by a wave of increased mitotic activity that peaked 5 days after the injection $(0.42 \pm$ $0 \cdot 055 \% ; n=6 ; P<0.001$ compared with gonadectomised rats at the start of treatment; Fig. 2A) before returning to levels seen in gonadectomised-only animals. In gonadectomised animals, but not in intact animals, the apoptotic index increased significantly over the 7-day period following injection of the testosterone esters (Fig. 2B). Injection of vehicle alone had no effects on mitotic index in either gonadectomised or intact rats (data not shown).

Treatment of intact animals with the same high-dose single bolus of testosterone esters increased anterior pituitary mitotic index by 3 days after the injection $(0 \cdot 19 \pm 0 \cdot 02 \% ; n=6 ; \quad P<0 \cdot 05$ compared with untreated controls) but without a detectable initial suppression of mitosis compared with intact controls (Fig. 3). When Sustanon treatment was delayed until 28 days after surgery, by which time the gonadectomy-induced wave of mitotic activity had passed, no initial suppression of mitosis was observed and the amplitude of the subsequent increase was similar to that found in intact rats, rising 4 days after the start of treatment to $0 \cdot 201 \pm 0 \cdot 037 \%$ $(n=5 ; P<0 \cdot 01$ compared with gonadectomised animals at the start of treatment) (Fig. 3). No concurrent changes in apoptotic activity were observed (data not shown). 


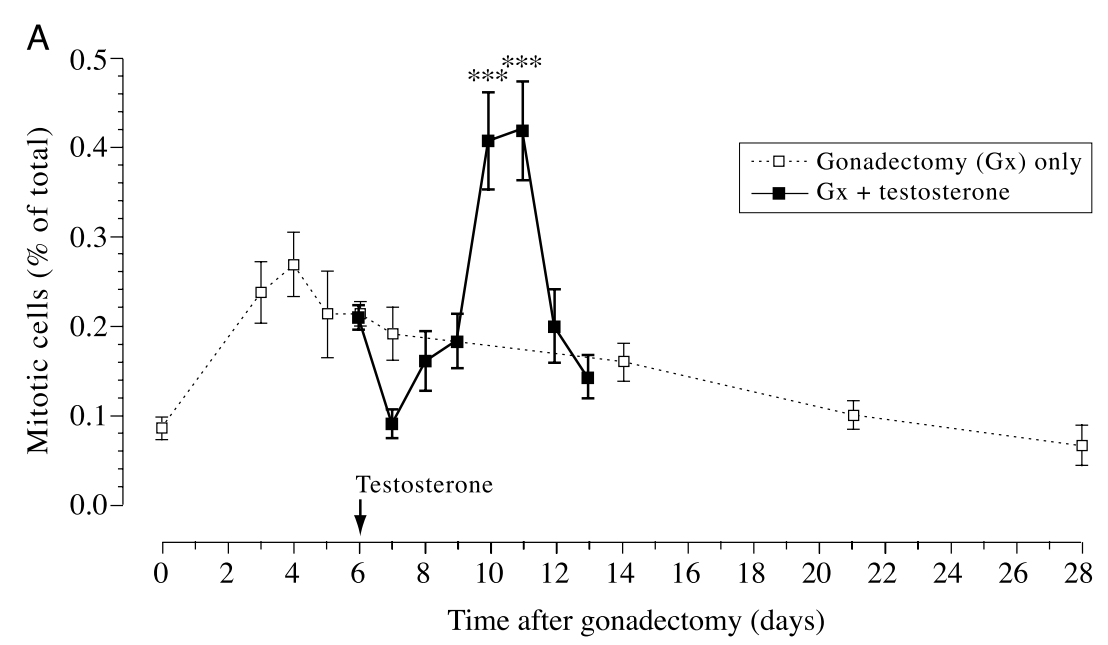

$\mathrm{B}$

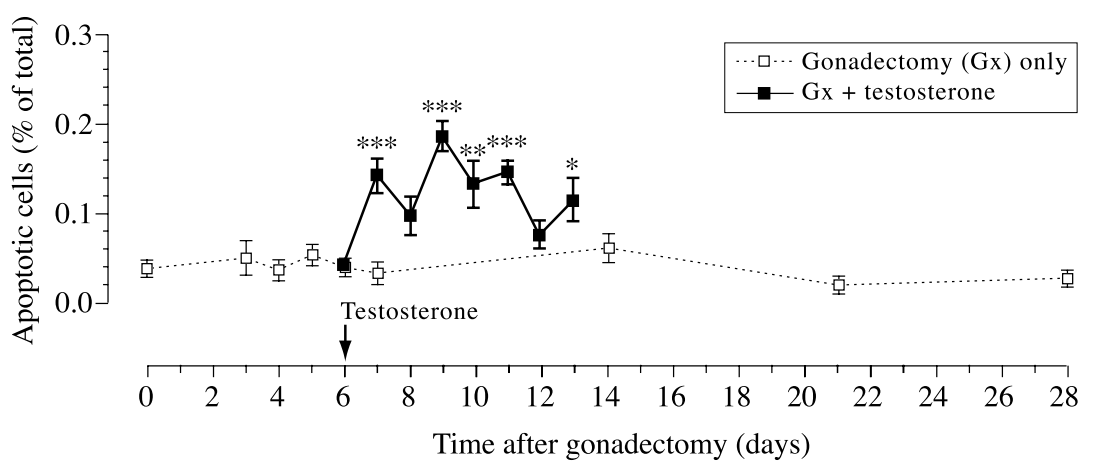

Figure 2 The effects of a single s.c. injection of Sustanon on anterior pituitary mitotic (A) and apoptotic (B) activity as a percentage of total parenchymal cells in male rats gonadectomised 6 days previously. Means \pm S.E. are shown. ${ }^{* * *} P<0 \cdot 001,{ }^{*} P<0 \cdot 01$ and ${ }^{*} P<0.05$ compared with gonadectomised-only animals on day 6 ( $n=6$ at all time points).

Division of the same total dose of testosterone esters into seven, once-daily s.c. injections beginning 6 days after gonadectomy resulted in a similar but slightly delayed pattern of anterior pituitary mitotic response (Fig. 4A). By 7 days after the start of treatment, the mitotic prevalence had reached $0 \cdot 337 \pm 0 \cdot 05 \%(n=6 ; P<0 \cdot 05$ compared with

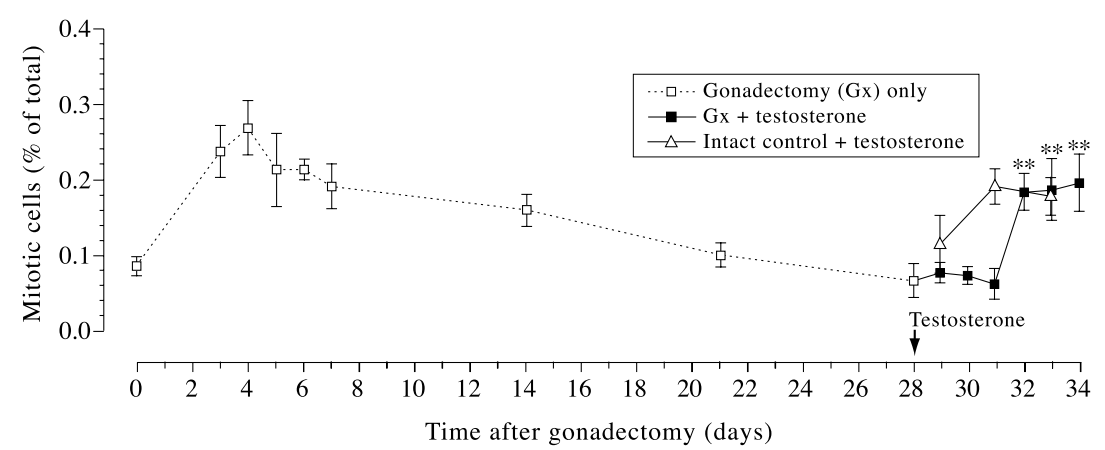

Figure 3 Changes in the prevalence of mitotic figures in the anterior pituitary of intact and gonadectomised rats following a single injection of Sustanon on day 28. Means \pm S.E. are shown. ${ }^{* *} P<0 \cdot 01$ compared with gonadectomised-only animals on day 28 ( $n=6$ at all time points). 

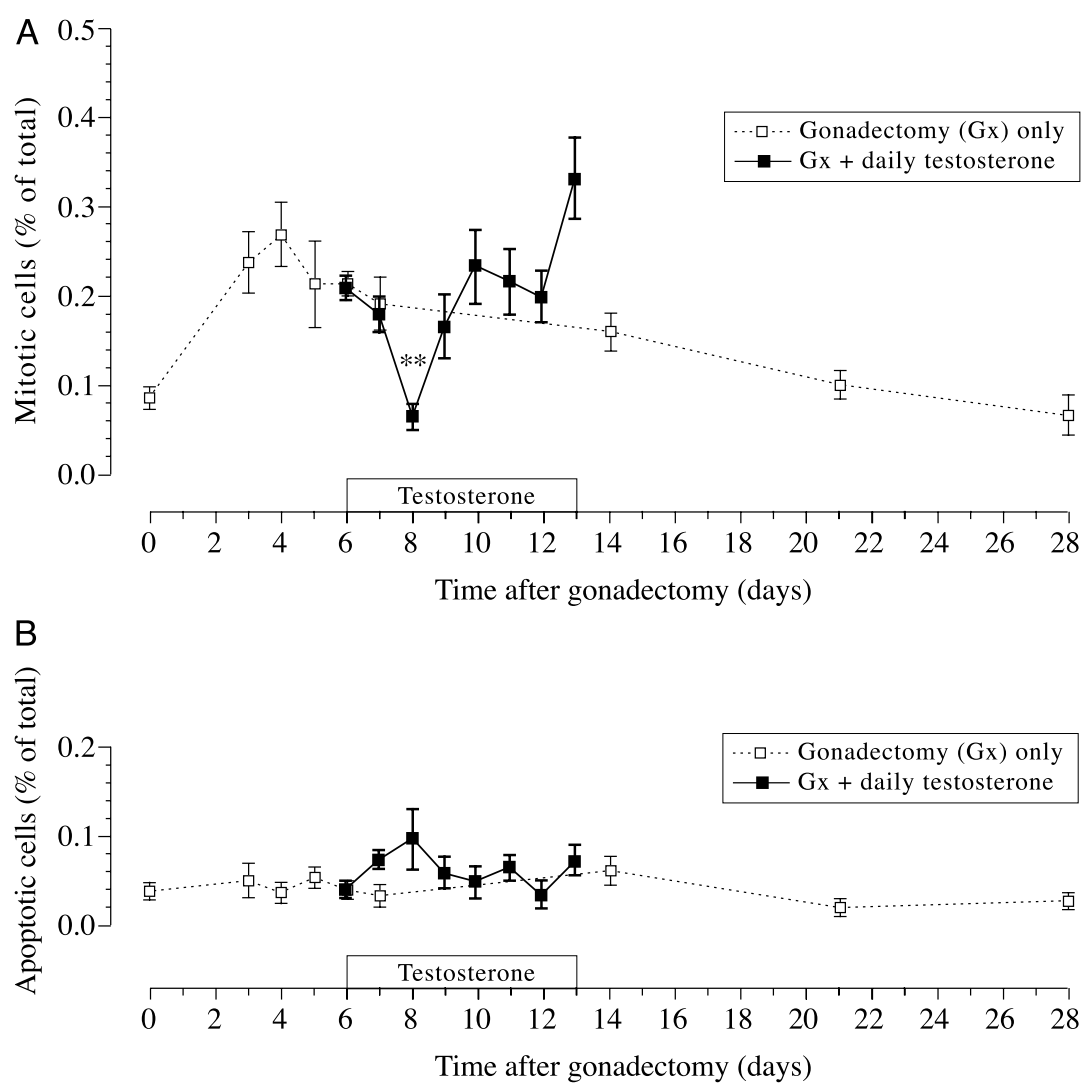

Figure 4 The effects of divided-dose s.c. injection of Sustanon on anterior pituitary mitotic (A) and apoptotic (B) activity in gonadectomised male rats. Means \pm S.E. are shown. ${ }^{* *} P<0.01$ and ${ }^{*} P<0.05$ compared with gonadectomised-only animals on day 6 ( $n=6$ at all time points).

gonadectomised-only animals at the start of treatment). A small increase in apoptotic activity was observed 2 days after the start of treatment but this did not reach statistical significance (Fig. 4B).

Substitution of Sustanon with daily s.c. injections of DHT from 6 days after gonadectomy significantly suppressed mitotic activity in the anterior pituitary throughout the treatment period compared with gonadectomised, but otherwise untreated, controls (Fig. 5). Treatment of intact animals with DHT also reduced the prevalence of mitotic figures compared with untreated controls although this did not reach statistical significance $(0 \cdot 054 \pm 0 \cdot 001 \%$ vs $0 \cdot 113 \pm 0 \cdot 015 \%$ on day 5 after the start of DHT treatment; $n=6$ ). No significant effects of DHT treatment were observed on apoptotic activity.

As expected, daily injections of oestradiol resulted in a highly significant increase in mitotic activity that peaked 3 days after the start of treatment in both gonadectomised $(0 \cdot 501 \pm 0 \cdot 065 \% ; n=6)$ and intact rats $(0 \cdot 432 \pm 0 \cdot 043 \%$; $n=6$ ) (Fig. 6) before returning to levels similar to those found in gonadectomised, but otherwise untreated, rats after 7 days. Again, there were no detectable effects of oestrogen treatment on the prevalence of apoptotic activity.

Concurrent daily injections of Sustanon with the nonsteroidal aromatase inhibitor letrozole - the latter starting 4 days after gonadectomy and $48 \mathrm{~h}$ before testosterone completely blocked the increase in mitotic activity seen with Sustanon alone in gonadectomised animals after 6-8 days of treatment (Fig. 7). Letrozole treatment alone had no significant effects on mitotic prevalence when compared with that measured in gonadectomised-only animals (Fig. 7A). No effects on apoptotic activity were observed (Fig. 7B).

\section{Discussion}

The present study suggests that circulating, testicularderived male gonadal hormones exert a tonic, inhibitory effect on anterior pituitary mitotic activity in young, adult male rats; either directly at the level of the pituitary and/or 

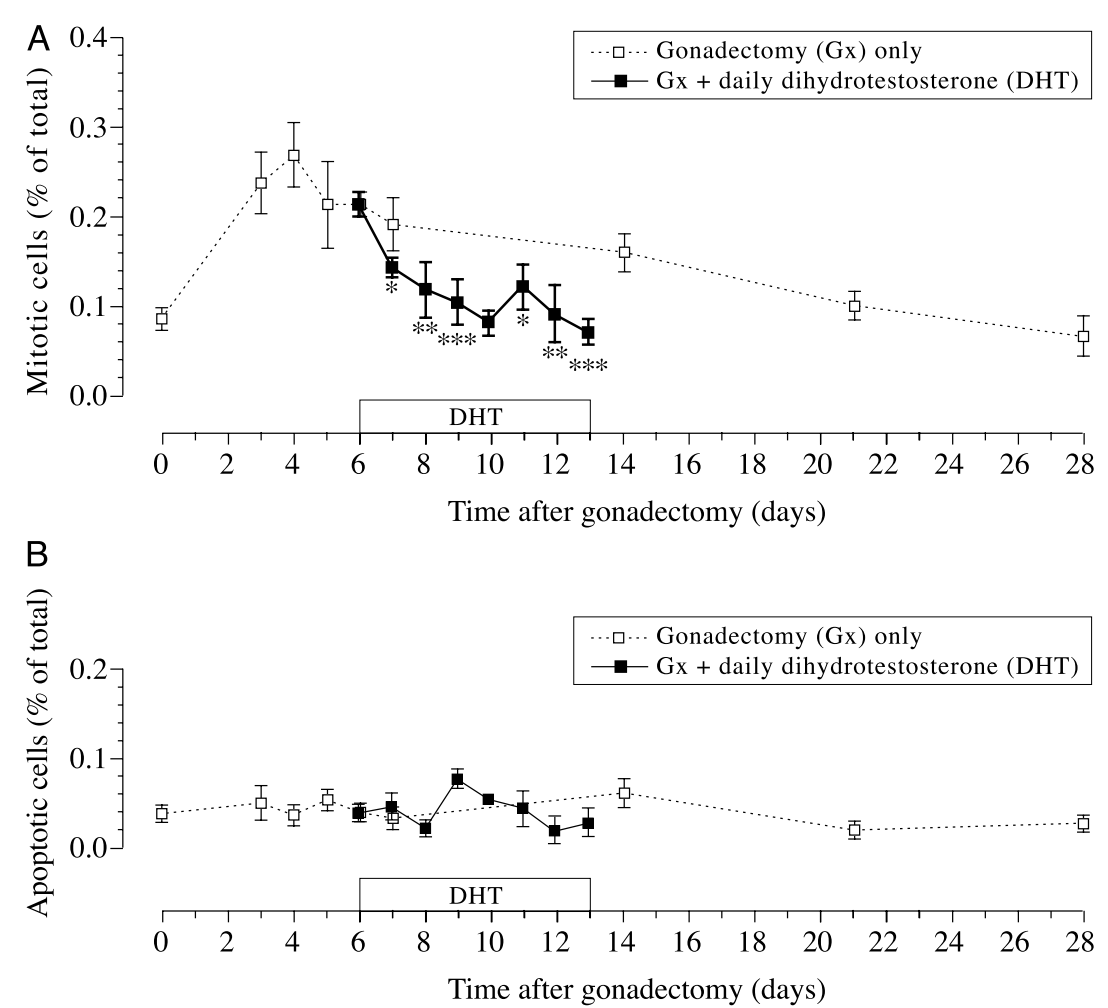

Figure 5 The effects of daily injections of DHT on anterior pituitary mitotic (A) and apoptotic (B) activity in rats gonadectomised 6 days previously. Means \pm S.E. are shown. ${ }^{* * *} P<0 \cdot 001,{ }^{* *} P<0 \cdot 01$ and ${ }^{*} P<0 \cdot 05$ compared with gonadectomised-only animals on day 6 ( $n=6$ at all time points).

indirectly via hypothalamic pathways. A pituitary mitotic response to gonadectomy has previously been reported (Sakuma et al. 1984, Inoue et al. 1985), as has a specific trophic effect of gonadotrophin-releasing hormone $(\mathrm{GnRH})$ on pituitary gonadotrophs both in vivo (Sakai et al. 1988) and in vitro (Childs \& Unabia 2001). The mitotic response to gonadectomy, which was not accompanied by any detectable change in apoptotic activity, was transient, with mitotic index returning to baseline levels within 28 days of surgery despite continued absence of circulating sex hormones. The self-limiting and transient nature of mitotic and apoptotic responses to persistent stimuli is a consistent and physiologically plausible characteristic of pituitary trophic activity, presumably mediated at least to some extent to receptor downregulation (Shupnik 2002). The mitotic response to adrenalectomy, for example, peaks within 2 or 3 days of surgery but returns to baseline levels within 2 weeks despite the continued absence of circulating glucocorticoids (Nolan et al. 1998). Our own data and those of others (Almeida et al. 1989) suggest that long-term gonadectomy and/or exposure to increased GnRH levels does not normally result in a persistent mitotic response in the anterior pituitary. In keeping with this, there are very few case reports of human pituitary adenomas secreting gonadotrophins developing after gonadal ablation (Nicolis et al. 1988); or of prolactinomas, for example, occurring in male patients receiving long-term, high-dose oestrogen (Gooren et al. 1988) or androgen treatment (Sodi et al. 2005). However, despite the return of the pituitary gland to normal in terms of the actual prevalence of mitotic events, we have previously shown that following longterm adrenalectomy with or without glucocorticoid treatments, trophic responses to subsequent stimuli are modified (Nolan \& Levy 2001, 2003). If this is also the case following exposure to altered sex hormone levels, then subtle and cumulative changes in specific cell populations may subsequently predispose the pituitary to adenoma induction or propagation.

Total changes in mitotic activity reached statistical significance at several time points after gonadectomy. It is tempting to speculate on the identity of the cells involved, but it is frustrating that even if an intrinsic marker such as a specific fluorescent tag were expressed in a defined cell population, potential changes in expression levels would still make accurate determination of relative and absolute cell numbers difficult and ultimately uninformative. The available alternatives for use in tissue sections - such as 


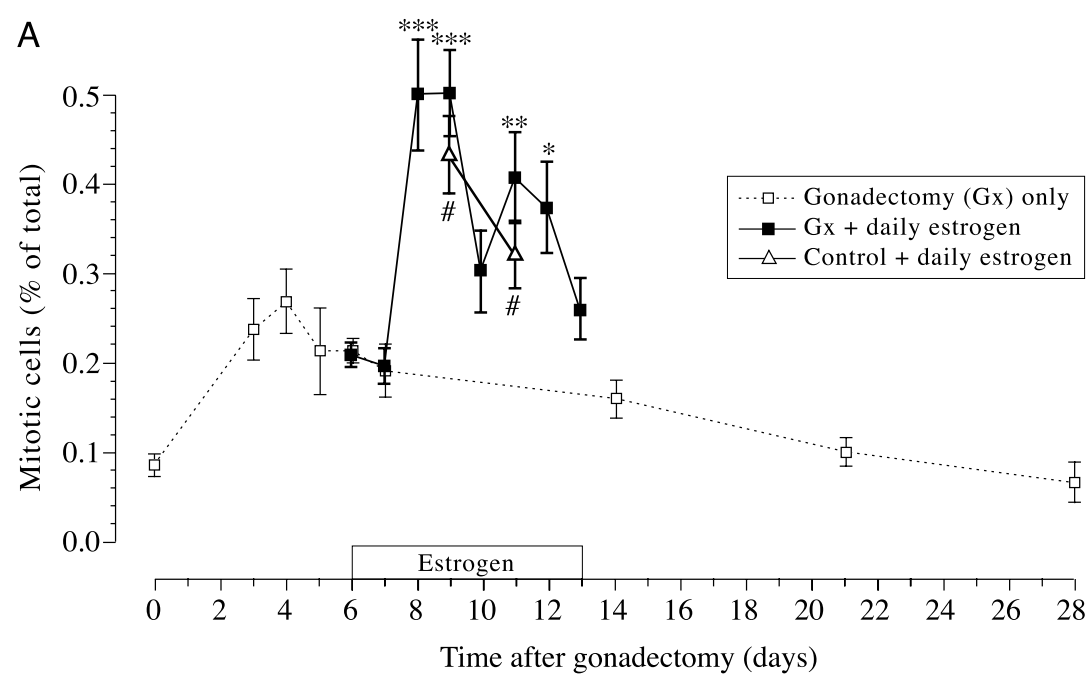

B

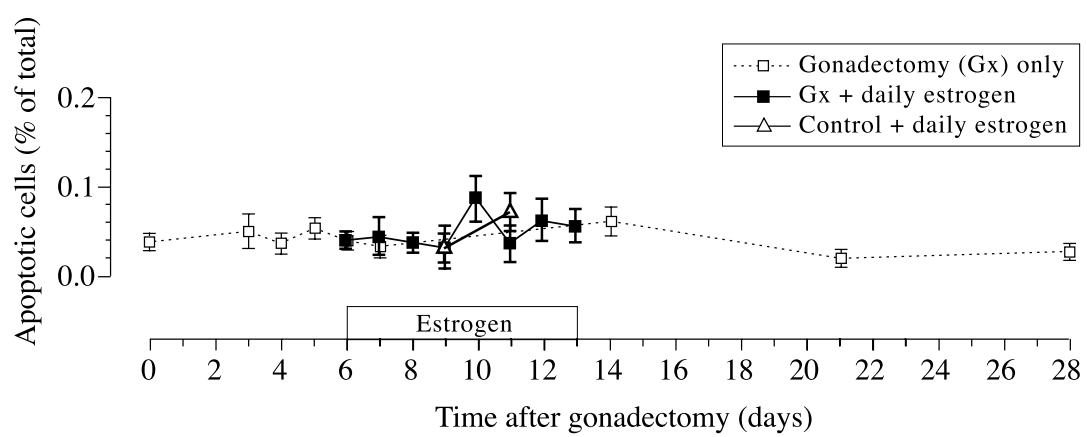

Figure 6 The effects of daily s.c. injections of oestrogen on anterior pituitary mitotic (A) and apoptotic (B) activity as a percentage of total parenchymal cells in male rats gonadectomised 6 days previously and in gonadal-intact rats. Means \pm S.E. are shown. ${ }^{* * *} P<0 \cdot 001,{ }^{* *} P<0 \cdot 01$ and ${ }^{*} P<0 \cdot 05$ compared with gonadectomised-only animals on day 6. ${ }^{\#} P<0.001$ compared with untreated gonadal-intact animals on day 0 ( $n=6$ at all time points).

TUNEL or detection of activated caspases for apoptosis, and detection of incorporated bromodeoxyuridine or Ki-67 for proliferating cells - depend on immunocytochemical methods that necessitate a more subjective and less definitive approach to the identification of positive cells than the methods employed in the current study, which are extremely well suited to comparative timecourse experiments. It seems probable, however - given known cross-talk between central neuroendocrine axes (Viau 2002, Chowen et al. 2004) and our own previous work on the nature of cells undergoing apoptosis in response to dexamethasone treatment after bilateral adrenalectomy (Nolan et al. 1998) - that in addition to mammotrophs and gonadotrophs (Sakuma et al. 1984, Inoue et al. 1985, Perez et al. 1986), several cell types including and perhaps predominated by apparently hormonally non-functioning populations are very likely to be involved.
A single, high-dose s.c. bolus of Sustanon or division of the dose into seven daily injections starting 6 days after gonadectomy, suppressed mitotic activity within $24-48 \mathrm{~h}$ compared with gonadectomised, vehicle-treated controls. By $72 \mathrm{~h}$, the mitotic index had returned to gonadectomised, vehicle-treated control levels but subsequently increased further to a statistically significant peak between 4 and 7 days after the start of treatment. In contrast, daily s.c. injections with DHT starting 6 days after gonadectomy, suppressed anterior pituitary mitotic activity throughout the course of treatment, reaching levels seen in intact, untreated animals within $48 \mathrm{~h}$. When daily s.c. injections of Sustanon were given concurrently with the aromatase inhibitor letrozole, the late increase in mitotic activity seen with Sustanon alone was completely abolished. Neither DHT treatment alone nor Sustanon treatment with letrozole, however, suppressed mitotic activity to below that seen in intact controls. Furthermore, 


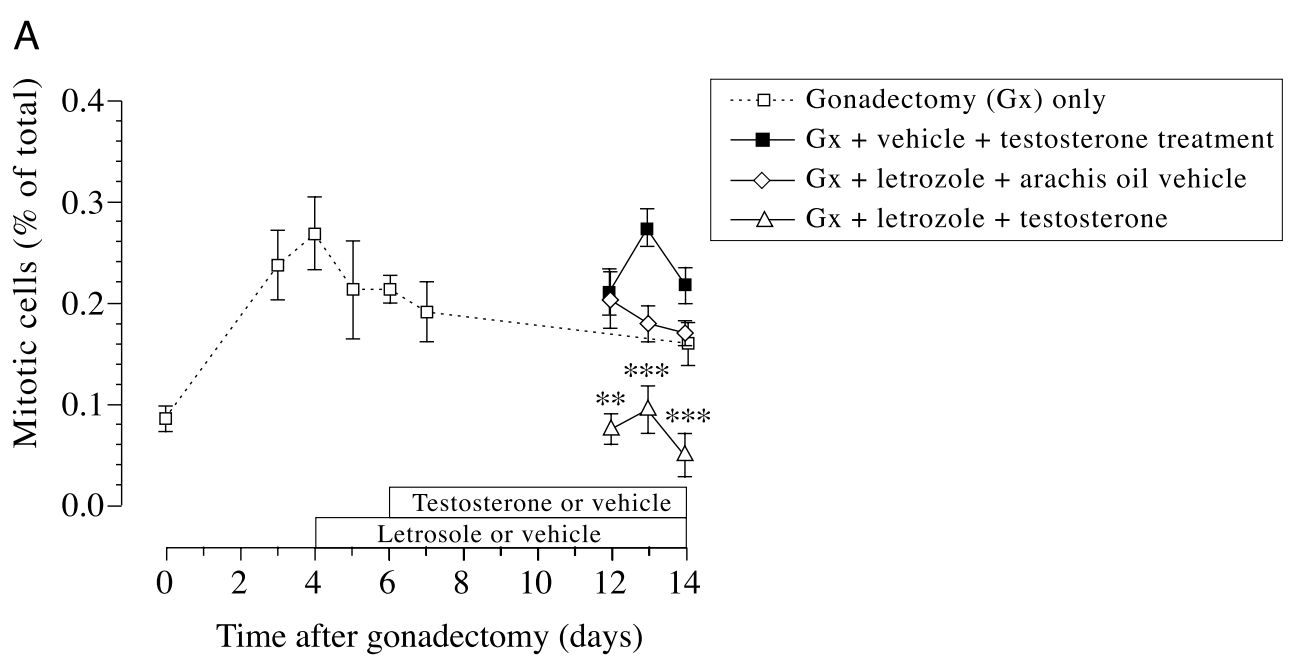

B

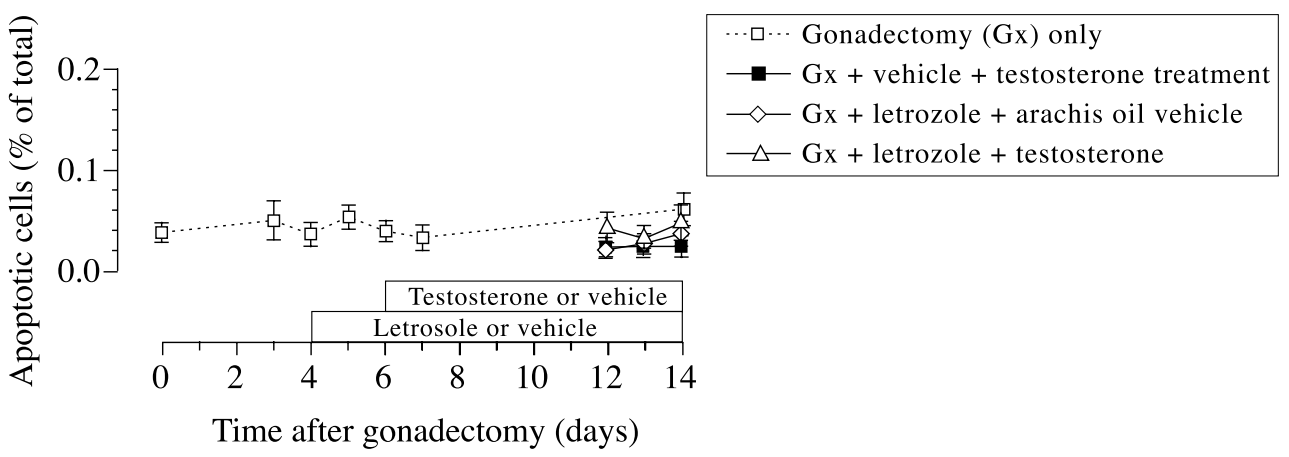

Figure 7 Changes in the prevalence of mitotic figures (A) and apoptotic bodies (B) in the anterior pituitary as a percentage of parenchymal cells in gonadectomised rats following daily injections of letrozole from day 4 and Sustanon from day 6 . Means \pm S.E. are shown. ${ }^{* *} P<0 \cdot 001$ and ${ }^{* *} P<0 \cdot 01$ compared with gonadectomised animals treated with testosterone only at each time point ( $n=6$ at all time points).

if Sustanon treatment was delayed until mitotic activity had returned to baseline, one month after gonadectomy, no further suppression of mitotic activity was seen. Daily administration of oestrogen in intact and gonadectomised animals produced a highly significant increase in mitotic activity within $72 \mathrm{~h}$ as expected. Together, these data strongly suggest that androgens facilitate inhibition of gonadectomy-enhanced anterior pituitary mitotic activity and that oestrogen, either exogenous or generated by local aromatisation of testosterone (Carretero et al. 2002), stimulates anterior pituitary mitotic activity.

With regards to apoptosis, a single, high-dose bolus of Sustanon induced an early increase in apoptotic activity in previously gonadectomised animals. The significance of this finding is not entirely clear as at lower daily doses, the slight early upward trend in apoptotic activity failed to reach significance at any time. However, this may be due to a problem of detection of extremely low numbers of events at any individual time point. It is probable that the absolute number of cells undergoing apoptosis is the same in both protocols but, because the timing of each individual apoptotic event is dependent on exposure to a critical concentration of Sustanon, this happens with a greater degree of synchronisation following a single large dose and results in significant detectable changes. No changes in apoptotic activity were detected with daily injections of DHT or with oestrogen treatment. It is also extremely difficult to detect significant suppression of baseline apoptotic activity. However, changes in apoptotic activity that are too small to verify statistically should not be overlooked, as these may produce very profound changes in cell populations (Nolan et al. 1998). Overall, these data suggest that androgens inhibit anterior pituitary mitotic activity and at very high doses may have the potential to increase apoptotic activity modestly.

An anti-proliferative function of androgens has been identified in the mammary epithelium of several mammalian species (Stege 2000, Zhou et al. 2000, Li et al. 2004), but the respective roles of androgen and oestrogen 
receptors in mediating trophic responses in the pituitary have not previously been clearly defined. Several different populations of pituitary cells, including subsets of lactotrophs and gonadotrophs, have been shown to express both androgen and oestrogen receptors (Pelletier 2000). Studies using the oestrogen receptor alpha knockout mouse (ERKO) (Lindzey et al. 1998), the aromatase knockout mouse (ArKO) (Fisher et al. 1998) and human aromatase $\mathrm{P} 450$ over-expressing transgenic mouse (AROM+) (Li et al. 2001) confirm that both androgen and oestrogen receptor pathways are important in the control of synthetic and secretory parameters in the hypothalamus and the pituitary. In addition to multiple abnormalities of reproductive function, the phenotype of the aromatase $\mathrm{P} 450$ over-expressing mouse includes pituitary hyperplasia, a defect that can be reversed in adulthood by treatment with an aromatase inhibitor (Li et al. 2004). Data from the present study suggest opposing roles for testosterone and oestrogen in mediating trophic responses in the anterior pituitary that are distinct from those mediating synthetic or secretory effects. The changes seen appear modest but support our previous work suggesting that there is considerable plasticity within the adult pituitary gland and that this might be relevant to subsequent hyperplastic and/or adenomatous changes.

In summary, we have shown that anterior pituitary mitotic activity is influenced by changes in circulating gonadal steroids following surgical gonadectomy and also by treatment with exogenous testosterone or oestrogen. Overt mitotic and apoptotic responses are transient and tend to return to baseline levels with time despite persistent changes in hormonal status. Testosterone suppresses the increase in mitosis induced by gonadectomy but is not able to depress baseline mitotic activity in intact rats or rats gonadectomised one month previously - by which time mitotic activity has returned to levels seen in intact animals. Oestrogen stimulates mitotic activity in the anterior pituitary at least in the short term. Lastly, the apparent stimulatory effect of testosterone on pituitary mitotic activity is likely to be the result of local aromatisation to oestrogen.

\section{Acknowledgements}

We would like to thank Dr Dean Evans, Novartis Pharma AG, Basel, Switzerland for supplying the letrozole.

\section{Funding}

This work was supported by a grant from The Wellcome Trust. The authors declare that there is no conflict of interest that would prejudice the impartiality of this scientific work.

\section{References}

Almeida OF, Hassan AH, Nikolarakis KE \& Martin GB 1989 Diminished role of LHRH in the control of gonadotroph morphology and function in the long-term castrated male rat. Journal of Endocrinology 123 263-273.

Brugal G, Dye R, Krief B, Chassery J-M, Tanke H \& Tucker JH 1992 HOME: Highly optimized microscope environment. Cytometry 13 109-116.

Carretero J, Burks DJ, Vazquez G, Rubio M, Hernandez E, Bodego P \& Vazquez R 2002 Expression of aromatase P450 is increased in spontaneous prolactinomas of aged rats. Pituitary 5 5-10.

Childs GV \& Unabia G 2001 Epidermal growth factor and gonadotropin-releasing hormone stimulate proliferation of enriched population of gonadotropes. Endocrinology 142 847-853.

Chowen JA, Frago LM \& Argente J 2004 The regulation of GH secretion by sex steroids. European Journal of Endocrinology 151 U95-U100.

Fisher CR, Graves KH, Parlow AF \& Simpson ER 1998 Characterization of mice deficient in aromatase (ArKO) because of targeted disruption of the cyp19 gene. PNAS 95 6965-6970.

Gooren U, Assies J, Asscheman H, de-Slegte R \& van-Kessel H 1988 Estrogen-induced prolactinoma in a man. Journal of Clinical Endocrinology and Metabolism 66 444-446.

Inoue K, Tanaka S \& Kurosumi K 1985 Mitotic activity of gonadotropes in the anterior pituitary of the castrated male rat. Cell and Tissue Research 240 271-276.

Levy A 2000 Is monoclonality in pituitary adenomas synonymous with neoplasia? Clinical Endocrinology 52 393-397.

Levy A 2002 Physiological implications of pituitary trophic activity. Journal of Endocrinology 174 147-155.

Levy A \& Lightman SL 2003 Molecular defects in the pathogenesis of pituitary tumors. Frontiers in Neuroendocrinology 24 94-127.

Li X, Nokkala E, Yan W, Streng T, Saarinen N, Warri A, Huhtaniemi I, Santti R, Makela S \& Poutanen M 2001 Altered structure and function of reproductive organs in transgenic male mice overexpressing human aromatase. Endocrinology 142 2435-2442.

Li X, Strauss L, Makela S, Streng T, Huhtaniemi I, Santti R \& Poutanen M 2004 Multiple structural and functional abnormalities in the $\mathrm{p} 450$ aromatase expressing transgenic male mice are ameliorated by a 450 aromatase inhibitor. American Journal of Pathology 164 1039-1048.

Lindzey J, Wetsel WC, Couse JF, Stoker T, Cooper R \& Korach KS 1998 Effects of castration and chronic steroid treatments on hypothalamic gonadotropin-releasing hormone content and pituitary gonadotropins in male wild-type and estrogen receptor-alpha knockout mice. Endocrinology 139 4092-4101.

Molitch ME 1997 Pituitary incidentalomas. Endocrinology and Metabolism Clinics of North America 26 725-740.

Nicolis G, Shimshi M, Allen C, Halmi NS \& Kourides IA 1988 Gonadotropin-producing pituitary adenoma in a man with long-standing primary hypogonadism. Journal of Clinical Endocrinology and Metabolism 66 237-241.

Nolan LA \& Levy A 2001 Anterior pituitary trophic responses to dexamethasone withdrawal and repeated dexamethasone exposures. Journal of Endocrinology 169 263-270.

Nolan LA \& Levy A 2003 Temporally sensitive trophic responsiveness of the adrenalectomized rat anterior pituitary to dexamethasone challenge: relationship between mitotic activity and apoptotic sensitivity. Endocrinology 144 212-219.

Nolan LA, Kavanagh E, Lightman SL \& Levy A 1998 Anterior pituitary cell population control: basal cell turnover and the effects of adrenalectomy and dexamethasone treatment. Journal of Neuroendocrinology 10 207-215. 
Nolan LA, Thomas CK \& Levy A 2004a Permissive effects of thyroid hormones on rat anterior pituitary mitotic activity. Journal of Endocrinology $18035-43$.

Nolan LA, Thomas CK \& Levy A $2004 b$ Pituitary mitosis and apoptotic responsiveness following adrenalectomy are independent of hypothalamic paraventricular nucleus CRH input. Journal of Endocrinology $181521-529$.

Pelletier G 2000 Localization of androgen and estrogen receptors in rat and primate tissues. Histology and Histopathology 15 1261-1270.

Perez RL, Machiavelli GA, Romano MI \& Burdman JA 1986 Prolactin release, oestrogens and proliferation of prolactin-secreting cells in the anterior pituitary gland of adult male rats. Journal of Endocrinology 108 399-403.

Sakai T, Inoue K, Hasegawa Y \& Karosumi K 1988 Effect of passive immunization to gonadotropin-releasing hormone $(\mathrm{GnRH})$ using $\mathrm{GnRH}$ anti-serum on the mitotic activity of gonadotrophs in castrated male rats. Endocrinology 122 2803-2808.

Sakuma S, Shirasawa N \& Yoshimura F 1984 A histometrical study of immunohistochemically identified mitotic adenohypophysial cells in immature and mature castrated rats. Journal of Endocrinology $100323-328$
Shupnik MA 2002 Oestrogen receptors, receptor variants and oestrogen actions in the hypothalamic-pituitary axis. Journal of Neuroendocrinology 14 85-94.

Sodi R, Fikri R, Diver M, Ranganath L \& Vora J 2005 Testosterone replacement-induced hyperprolactinaemia: case report and review of the literature. Annals of Clinical Biochemistry 42 153-159.

Stefaneanu L 1997 Pituitary sex steroid receptors: localization and function. Endocrine Pathology 8 91-108.

Stege R 2000 Potential side-effects of endocrine treatment of long duration in prostate cancer. Prostate Supplement $1038-42$.

Viau V 2002 Functional cross-talk between the hypothalamic-pituitary-gonadal and -adrenal axes. Journal of Neuroendocrinology 14 506-513.

Zhou J, Ng S, Adesanya-Famuiya O, Anderson K \& Bondy CA 2000 Testosterone inhibits estrogen-induced mammary epithelial proliferation and suppresses estrogen receptor expression. FASEB Journal 14 1725-1730.

Received 10 November 2005

Accepted 16 November 2005 